\title{
Correction
}

\section{Correction: Mitochondrionopathy Phenotype in Doxorubicin-Treated Wistar Rats Depends on Treatment Protocol and Is Cardiac-Specific}

\section{The PLOS ONE Staff}

\section{Notice of Republication}

This article was republished on April 24, 2014, to correct errors in in-text references and the missing Figure 4 in the PDF version of the article. Please download this article again to view the correct version. The originally published, uncorrected article and the republished, corrected article are provided here for reference.

\section{Supporting Information}

File S1. Originally published, uncorrected article.

File S2. Republished corrected article.

\section{Reference}

1. Pereira GC, Pereira SP, Pereira GV, Lumini JA, Magalhães J, et al. (2012) Mitochondrionopathy Phenotype in Doxorubicin-Treated Wistar Rats Depends on Treatment Protocol and Is Cardiac-Specific. PLoS ONE 7(6): e38867. doi:10.1371/journal.pone.0038867
Citation: The PLOS ONE Staff (2014) Correction: Mitochondrionopathy Phenotype in Doxorubicin-Treated Wistar Rats Depends on Treatment Protocol and Is Cardiac-Specific. PLoS ONE 9(5): e97795. doi:10.1371/journal.pone.0097795

Published May 9, 2014

Copyright: (๑) 2014 The PLOS ONE Staff. This is an open-access article distributed under the terms of the Creative Commons Attribution License, which permits unrestricted use, distribution, and reproduction in any medium, provided the original author and source are credited. 